\title{
DIRECT METHODS IN GEOMETRICAL OPTICS
}

\author{
BY \\ M. HERZBERGER
}

Communication no. 851 from the Kodak Research Laboratories

This paper presents a different approach to the problems of geometrical optics, in order to attack some problems hitherto insoluble in practice.

The investigation has been restricted to rotationally symmetric optical systems for practical reasons only; the procedures can be applied to the general case. Let us choose two rectangular coordinate systems such that the $z$ - and $z^{\prime}$-axes coincide with the optical axis, the $y$-and $y^{\prime}$-, and $x$ - and $x^{\prime}$-axes being, respectively, parallel. An object ray is then defined by its intersection point with the plane $z=0$; (vector $\vec{a}$, coordinates $x, y, 0$ ) and by the vector $\vec{s}$ of length $n$ ( $n$ refractive index) along the ray (coordinates $\left.\xi, \eta, \zeta=\left(n^{2}-\xi^{2}-\eta^{2}\right)^{1 / 2}\right)$; the image is given by $a^{\prime \rightarrow}\left(x^{\prime}, y^{\prime}, 0\right)$ and $s^{\prime \rightarrow}\left(\xi^{\prime}, \eta^{\prime}, \zeta^{\prime}=\left(n^{\prime 2}-\xi^{\prime 2}-\eta^{\prime 2}\right)^{1 / 2}\right)$, with $n^{\prime}$ the refractive index of the image space.

The problem of the optical designer is to find the image ray if the object ray is given, or in other words, to compute, for a given optical system, $x^{\prime}$, $y^{\prime}, \xi^{\prime}$, and $\eta^{\prime}$ as functions of $x, y, \xi$, and $\eta$. If this problem is solved for a single surface and arbitrary positions of the object and image planes, it is solved for any rotationally symmetrical optical system merely by making a succession of substitutions. To solve the problem for a single surface, which will here be assumed to be a spherical surface, we first place object and image planes at the center of the refracting surface, and then calculate the functions. Having done this, we have only to compute the intersection points of the image rays with a parallel plane through another origin, a simple geometric problem. We can use this method for the manifold of all rays in a procedure similar to the ordinary way of tracing meridional rays; and applied to an individual ray, it becomes a straightforward method for tracing skew rays through an optical system.

\section{General Formulae}

Before deriving these equations, we shall inspect the general conditions of optical image formation $\left({ }^{1}\right)$. Because of the rotational symmetry we can write

Presented to the Society, October 31, 1942; received by the editors April 17, 1942.

(1) The term "optical image formation" refers to the one-to-one correspondence of object and image rays in an optical system. Not every one-to-one correspondence can be considered as an optical image formation. The conditions are derived in this paper: For the mathematician it might be noted that an optical image formation is a special kind of contact transformation. 


$$
\begin{array}{ll}
x^{\prime}=A x+B \xi, & \xi^{\prime}=C x+D \xi \\
y^{\prime}=A y+B \eta, & \eta^{\prime}=C y+D \eta
\end{array}
$$

in which $A, B, C$, and $D$ are functions of $u_{1}, u_{2}$, and $u_{3}$, which are symmetric functions of $x, y, \xi$, and $\eta$ :

$$
\begin{aligned}
& u_{1}=(1 / 2)\left(x^{2}+y^{2}\right), \\
& u_{2}=x \xi+y \eta \\
& u_{3}=(1 / 2)\left(\xi^{2}+\eta^{2}\right) .
\end{aligned}
$$

However, $A, B, C$, and $D$ are not arbitrary functions of $u_{1}, u_{2}$, and $u_{3}$. Our first task is to derive the differential equations connecting them.

According to the fundamental optical invariant $\left({ }^{2}\right)$, for any two-parameter manifolds (parameters $u, v$ ), we have (abbreviating $\partial \vec{s} / \partial u=\vec{u}$ ),

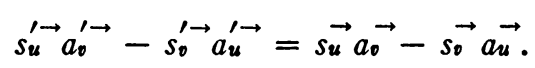

Taking the following variables in turn for $u$ and $v: x, y ; x, \xi ; x, \eta ; y, \xi ; y, \eta$; and $\xi, \eta$ and turning from the vectors to the coordinates, we find that

$$
\begin{aligned}
& \left(x_{x}^{\prime} \xi_{y}^{\prime}+y_{x}^{\prime} \eta_{y}^{\prime}\right)-\left(x_{y}^{\prime} \xi_{x}^{\prime}+y_{y}^{\prime} \eta_{x}^{\prime}\right)=0, \\
& \left(x_{x}^{\prime} \xi_{\xi}^{\prime}+y_{x}^{\prime} \eta_{\xi}^{\prime}\right)-\left(x_{\xi}^{\prime} \xi_{x}^{\prime}+y_{\xi}^{\prime} \eta_{x}^{\prime}\right)=1,
\end{aligned}
$$

Writing $A_{\nu}$ for $\partial A / \partial u_{\nu}$, and so forth, we now obtain from (1) and (2),

$$
\begin{aligned}
& x_{x}^{\prime}=A+A_{1} x^{2}+\left(A_{2}+B_{1}\right) x \xi+B_{2} \xi^{2}, \\
& x_{\nu}^{\prime}=A_{1} x y+A_{2} x \eta+B_{1} \xi y+B_{2} \xi \eta,
\end{aligned}
$$

Inserting (5) in (4), we obtain the equations

(6)

$$
\begin{aligned}
(x \eta-y \xi) I & =0, \\
(A D-B C)+x^{2} I+x \xi I I+\xi^{2} I I I & =1, \\
x y I+x \eta I I+\xi \eta I I I & =0, \\
x y I+y \xi I I+\xi \eta I I I & =0, \\
(A D-B C)+y^{2} I+y \eta I I+\eta^{2} I I I & =1, \\
(x \eta-y \xi) I I I & =0 ;
\end{aligned}
$$

(2) For the historical background of formula (3) and its connection with different branches of mathematics, the reader is referred to $\mathrm{M}$. Herzberger, Theory of transversal curves and the connections between the calculus of variations and the theory of partial differential equations, Proc. Nat. Acad. Sci. U.S.A. vol. 24 (1938) pp. 466-473. 
where

$$
\begin{aligned}
I= & A C_{2}+D A_{1}-C A_{2}-B C_{1}+2 u_{1}\left(A_{1} C_{2}-A_{2} C_{1}\right) \\
& +u_{2}\left(A_{1} D_{2}-A_{2} D_{1}+B_{1} C_{2}-B_{2} C_{1}\right)+2 u_{3}\left(B_{1} D_{2}-B_{2} D_{1}\right), \\
I I= & A C_{3}+D B_{1}-C A_{3}-B D_{1}+2 u_{1}\left(A_{1} C_{3}-A_{3} C_{1}\right) \\
& +u_{2}\left(A_{1} D_{3}-A_{3} D_{1}+B_{1} C_{3}-B_{3} C_{1}\right)+2 u_{3}\left(B_{1} D_{3}-B_{3} D_{1}\right), \\
I I I= & A D_{3}+D B_{2}-C B_{3}-B D_{2}+2 u_{1}\left(A_{2} C_{3}-A_{3} C_{2}\right) \\
& +u_{2}\left(A_{2} D_{3}-A_{3} D_{2}+B_{2} C_{3}-B_{3} C_{2}\right)+2 u_{3}\left(B_{2} D_{3}-B_{3} D_{2}\right) .
\end{aligned}
$$

Equations (6) can be satisfied for all rays only if

$$
\begin{gathered}
I=I I=I I I=0 ; \\
A D-B C=1 .
\end{gathered}
$$

The fulfillment of the four equations (8) is necessary and sufficient to guarantee that equation (1) determines an optical image formation. We can of course eliminate one of the functions, for example, $D$, and have three functions $A, B$, and $C$, and three differential equations connecting them. We find that

$$
D=\frac{1+B C}{A},
$$

$$
D_{\nu}=\frac{C}{A} B_{\nu}+\frac{B}{A} C_{\nu}-\frac{1+B C}{A^{2}} A_{\nu}
$$

Inserting this in (7), we obtain these equations:

$$
\begin{aligned}
\left(A C_{2}-C A_{2}\right) & +\frac{B}{A}\left(C A_{1}-A C_{1}\right)+\frac{A_{1}}{A}+\left(A_{1} C_{2}-A_{2} C_{1}\right)\left(2 u_{1}+\frac{B}{A} u_{2}\right) \\
& +\left(A_{1} B_{2}-A_{2} B_{1}\right)\left(\frac{C}{A} u_{2}+2 u_{3} \frac{1+B C}{A^{2}}\right) \\
& +\left(B_{1} C_{2}-B_{2} C_{1}\right)\left(u_{2}+2 u_{3} \frac{B}{A}\right)=0 \\
\left(A C_{3}-C A_{3}\right) & +\frac{B^{2}}{A}\left(C A_{1}-A C_{1}\right)+\frac{B A_{1}+A B_{1}}{A^{2}} \\
& +\left(A_{1} C_{3}-A_{3} C_{1}\right)\left(2 u_{1}+\frac{B}{A} u_{2}\right) \\
& +\left(A_{1} B_{3}-A_{3} B_{1}\right)\left(\frac{C}{A} u_{2}+2 u_{3} \frac{1+B C}{A^{2}}\right) \\
& +\left(B_{1} C_{3}-B_{3} C_{1}\right)\left(u_{2}+2 u_{3} \frac{B}{A}\right)=0
\end{aligned}
$$




$$
\begin{aligned}
\frac{B}{A}\left(A C_{3}-C A_{3}\right) & +\frac{B^{2}}{A^{2}}\left(C A_{2}-A C_{2}\right)+\frac{B A_{2}+A B_{2}}{A^{2}}-\frac{A_{3}}{A} \\
& +\left(A_{2} C_{3}-A_{3} C_{2}\right)\left(2 u_{1}+\frac{B}{A} u_{2}\right) \\
& +\left(A_{2} B_{3}-A_{3} B_{2}\right)\left(\frac{C}{A} u_{2}+2 u_{3} \frac{1+B C}{A^{2}}\right) \\
& +\left(B_{2} C_{3}-B_{3} C_{2}\right)\left(u_{2}+2 u_{3} \frac{B}{A}\right)=0 .
\end{aligned}
$$

Let us multiply these equations by $A_{3},-A_{2}, A_{1} ; B_{3},-B_{2}, B_{1} ; C_{3},-C_{2}, C_{1}$, respectively, and add. We thus obtain three new equations, which can replace equations (10) if

$$
\left|\begin{array}{lll}
A_{1} & A_{2} & A_{3} \\
B_{1} & B_{2} & B_{3} \\
C_{1} & C_{2} & C_{3}
\end{array}\right|=\Delta \neq 0
$$

that is, $A, B$, and $C$ are three independent functions. (In this case we can construct their inverse functions, that is, we can calculate $u_{1}, u_{2}$, and $u_{3}$ as functions of $A, B$, and $C$. We shall make use of this later.) In either case, we obtain from (10) three equations:

$$
\begin{aligned}
A\left(C_{2} A_{3}-C_{3} A_{2}\right) & +B\left(A_{1} C_{3}-C_{1} A_{3}\right)+\frac{B^{2}}{A}\left(C_{1} A_{2}-C_{2} A_{1}\right) \\
& +\frac{1}{A}\left(B_{2} A_{1}-B_{1} A_{2}\right)+\left(u_{2}+2 u_{3} \frac{B}{A}\right) \Delta=0, \\
A\left(C_{2} B_{3}-C_{3} B_{2}\right)+ & B\left(B_{1} C_{3}-C_{1} B_{3}\right)+C\left(A_{3} B_{2}-A_{2} B_{3}\right) \\
& +\frac{1+B C}{A}\left(A_{1} B_{3}-B_{1} A_{3}\right) \\
& +\frac{B}{A}\left(\frac{1+B C}{A}\right)\left(A_{2} B_{1}-A_{1} B_{2}\right)+\frac{B^{2}}{A}\left(C_{1} B_{2}-C_{2} B_{1}\right) \\
& +\left(2 u_{1}+\frac{B}{A} u_{2}\right) \Delta=0, \\
C\left(C_{2} A_{3}-C_{3} A_{2}\right) & +\frac{1+B C}{A}\left(A_{1} C_{3}-C_{1} A_{3}\right) \\
& +\frac{B}{A}\left(A_{2} C_{1}-A_{1} C_{2}\right)\left(\frac{1+B C}{A}\right)+\frac{1}{A}\left(B_{2} C_{1}-B_{1} C_{2}\right) \\
& +\left(\frac{C}{A} u_{2}+2 u_{3} \frac{1+B C}{A^{2}}\right) \Delta=0 .
\end{aligned}
$$


These equations are equivalent to:

$$
\begin{aligned}
A\left(A_{1} C_{3}-A_{8} C_{1}\right)+B\left(A_{2} C_{1}-A_{1} C_{2}\right)+ & C\left(A_{2} B_{1}-A_{1} B_{2}\right) \\
& +A\left(B_{2} C_{1}-B_{1} C_{2}\right)+2 u_{3} \Delta=0, \\
A\left(A_{3} C_{2}-A_{2} C_{3}\right)+ & \frac{1+B C}{A}\left(A_{2} B_{1}-A_{1} B_{2}\right)-B\left(B_{2} C_{1}-B_{1} C_{2}\right)+u_{2} \Delta=0, \\
A\left(B_{3} C_{2}-C_{3} B_{2}\right)+ & B\left(B_{1} C_{3}-C_{1} B_{3}\right)+C\left(A_{3} B_{2}-A_{2} B_{3}\right) \\
- & B\left(A_{3} C_{2}-C_{3} A_{2}\right)+\frac{1+B C}{A}\left(A_{1} B_{3}-B_{1} A_{3}\right) \\
+ & 2 u_{1} \Delta=0 .
\end{aligned}
$$

If $\Delta$ is not identically equal to zero, we now get very simple equations for the inverse functions, if we insert

$$
\begin{array}{lll}
\frac{\partial u_{1}}{\partial A}=\frac{B_{2} C_{3}-B_{3} C_{2}}{\Delta}, & \frac{\partial u_{1}}{\partial B}=\frac{A_{3} C_{2}-A_{2} C_{3}}{\Delta}, & \frac{\partial u_{1}}{\partial C}=\frac{A_{2} B_{3}-A_{3} B_{2}}{\Delta}, \\
\frac{\partial u_{2}}{\partial A}=\frac{B_{1} C_{3}-B_{3} C_{1}}{\Delta}, & \frac{\partial u_{2}}{\partial B}=\frac{A_{1} C_{3}-A_{3} C_{1}}{\Delta}, & \frac{\partial u_{2}}{\partial C}=\frac{A_{3} B_{1}-A_{1} B_{3}}{\Delta}, \\
\frac{\partial u_{8}}{\partial A}=\frac{B_{1} C_{2}-B_{2} C_{1}}{\Delta}, & \frac{\partial u_{3}}{\partial B}=\frac{A_{2} C_{1}-A_{1} C_{2}}{\Delta}, & \frac{\partial u_{2}}{\partial C}=\frac{A_{1} B_{2}-A_{2} B_{1}}{\Delta} .
\end{array}
$$

Thus, equations (13) give finally

$$
\begin{array}{r}
A \frac{\partial u_{2}}{\partial B}+B \frac{\partial u_{3}}{\partial B}-C \frac{\partial u_{3}}{\partial C}-A \frac{\partial u_{3}}{\partial A}+2 u_{3}=0 \\
A \frac{\partial u_{1}}{\partial B}-\frac{1+B C}{A} \cdot \frac{\partial u_{3}}{\partial C}+B \frac{\partial u_{3}}{\partial A}+u_{2}=0 \\
A \frac{\partial u_{1}}{\partial A}-B \frac{\partial u_{2}}{\partial A}+C \frac{\partial u_{1}}{\partial C}+B \frac{\partial u_{1}}{\partial B}+\frac{1+B C}{A} \cdot \frac{\partial u_{2}}{\partial C}-2 u_{1}=0
\end{array}
$$

as fundamental equations for optical image formation.

\section{A SPECIAL KIND OF OPTICAL IMAGE FORMATION}

As an example, let us consider a special case in which $\Delta \equiv 0$. Let us assume $B \equiv 0$; then

$$
\begin{array}{ll}
x^{\prime}=A x, & \xi^{\prime}=C x+(1 / A) \xi, \\
y^{\prime}=A y, & \eta^{\prime}=C y+(1 / A) \eta .
\end{array}
$$

This kind of image formation is of great importance in optics. A single 
sphere produces this type of image; so does a system of concentric surfaces, a so-called concentric lens system. On the other hand, the attempt to realize the dream of an optical designer, a system which gives a sharp image of one plane $z=0$ upon another $\left(z^{\prime}=0\right)$, leads to an image formation given by formula (16) with the special condition that $A$ is a constant.

In general, $A$ must be different from 0 and, inserting $B=B_{1}=B_{2}=B_{3}=0$, equations (10) give

$$
\begin{array}{r}
\left(A C_{2}-C A_{2}\right)+\left(A_{1} / A\right)+\left(A_{1} C_{2}-A_{2} C_{1}\right) 2 u_{1}=0, \\
\left(A C_{3}-C A_{3}\right)+\left(A_{1} C_{3}-A_{3} C_{1}\right) 2 u_{1}=0, \\
-\left(A_{3} / A\right)+\left(A_{2} C_{3}-A_{3} C_{2}\right) 2 u_{1}=0,
\end{array}
$$

as differential equations for the two functions. Equations (12) give

$$
A\left(C_{2} A_{3}-C_{3} A_{2}\right)=0 \text { and } C\left(C_{2} A_{3}-C_{3} A_{2}\right)+\frac{1}{A}\left(A_{1} C_{3}-C_{1} A_{3}\right)=0,
$$

or

$$
C_{2} \dot{A}_{3}-C_{3} A_{2}=A_{1} C_{3}-A_{3} C_{1}=0 .
$$

Inserting (19) in (17), we find that

$$
\begin{gathered}
A_{3}=C_{3}=0, \\
\left(A C_{2}-C A_{2}\right)+\left(A_{1} / A\right)+\left(A_{1} C_{2}-A_{2} C_{1}\right) 2 u_{1}=0 .
\end{gathered}
$$

We see that in this case $A$ and $C$ are functions of $u_{1}$ and $u_{2}$ alone. We introduce $D=1 / A$ and find, as differential equations for $C$ and $D$,

$$
\begin{gathered}
D_{3}=C_{3}=0, \\
D C_{2}+C D_{2}-D D_{1}+\left(C_{1} D_{2}-D_{1} C_{2}\right) 2 u_{1}=0 .
\end{gathered}
$$

In the special case of $D=$ const., $C_{2}=0$. That means $C$ is a function of $u_{1}$ alone. We have

$$
\begin{array}{ll}
x^{\prime}=A_{0} x, & \xi^{\prime}=C\left(u_{1}\right) x+\left(1 / A_{0}\right) \xi, \\
y^{\prime}=A_{0} y, & \eta^{\prime}=C\left(u_{1}\right) y+\left(1 / A_{0}\right) \eta .
\end{array}
$$

Equation (22) is a generalization of the well known sine condition of Abbe; $A_{0}$ is the magnification of the image and $(22)_{2}$ can be written in the familiar form

$$
A_{0} \xi^{\prime}-\xi=C\left(u_{1}\right) x, \quad A_{0} \eta^{\prime}-\eta=C\left(u_{1}\right) y,
$$

where the right sides of equations (23) are independent of $\xi$ and $\eta$.

Let us now investigate the general case, in which $C$ and $D$ are independent functions of $u_{1}$ and $u_{2}$, such that 


$$
C_{1} D_{2}-C_{2} D_{1} \neq 0 \text {. }
$$

Here we may again construct the inverse functions, and consider $u_{1}$ and $u_{2}$ as functions of $C$ and $D$. Then we have

$$
\begin{aligned}
\frac{\partial u_{1}}{\partial C}=\frac{D_{2}}{C_{1} D_{2}-C_{2} D_{1}}, & \frac{\partial u_{1}}{\partial D} & =\frac{-C_{2}}{C_{1} D_{2}-C_{2} D_{1}}, \\
\frac{\partial u_{2}}{\partial C}=\frac{-D_{1}}{C_{1} D_{2}-C_{2} D_{1}}, & \frac{\partial u_{2}}{\partial D} & =\frac{C_{1}}{C_{1} D_{2}-C_{2} D_{1}} .
\end{aligned}
$$

Inserting (25) in (21) gives

$$
C \frac{\partial u_{1}}{\partial C}-D \frac{\partial u_{1}}{\partial D}+D \frac{\partial u_{2}}{\partial C}+2 u_{1}=0 .
$$

The solution of this differential equation would solve our problem.

\section{THE SINGLE SPHERICAL SURFACE}

Again we attack a special case of the aforesaid problem, namely, the single refracting spherical surface. The coordinate origins in the object and image spaces are placed at the center of the sphere, the $x$ - and $x^{\prime}-, y$ - and $y^{\prime}$-axes coinciding. Let $a^{\rightarrow}\left(a^{\prime \rightarrow}\right)$ be the vector $(x, y, 0)\left(x^{\prime}, y^{\prime}, 0\right)$, respectively, to the intersection point of the ray with the coordinate plane $z\left(z^{\prime}\right)=0$; let $\vec{s}(\xi, \eta, \zeta)$, $s^{\prime \prime}\left(\xi^{\prime}, \eta^{\prime}, \zeta^{\prime}\right)$, respectively, be the vectors of length $n\left(n^{\prime}\right)$ along the object and image rays.

Let $r$ be the radius of the refracting surface, positive if the surface is convex with respect to the direction of the light ray, and negative if the surface is concave. Let $r$ be the vector of length $r$ along the incidence normal. The refraction law can then be written

$$
s \rightarrow \times r \rightarrow=s^{\prime} \rightarrow \times r \rightarrow \text {, or } \quad s^{\prime} \rightarrow-s \rightarrow=C r .
$$

The coordinates are then given by equations (2):

$$
\begin{aligned}
& u_{1}=\left(x^{2}+y^{2}\right) / 2=a^{2} \rightarrow / 2, \\
& u_{2}=x \xi+y \eta=a \rightarrow \cdot s \rightarrow .
\end{aligned}
$$

Two values, $\lambda$ and $\lambda^{\prime}$, exist such that

$$
a \rightarrow+\lambda s^{\prime}=r \rightarrow=a^{\prime} \rightarrow+\lambda^{\prime} s^{\prime} \rightarrow,
$$

and $a^{\prime \rightarrow}$ and $a^{\rightarrow}$ must have the same direction, since they both lie in the intersection line of the incidence plane with the plane $z=0$. From (29) and (27) we find that

$$
a^{\prime} \rightarrow \times s^{\prime} \rightarrow=r \rightarrow \times s^{\prime} \rightarrow=r \rightarrow \times s \rightarrow=a \rightarrow \times s \rightarrow .
$$


This gives finally

$$
\begin{aligned}
& a^{\prime} \rightarrow=(1 / D) a \rightarrow, \\
& s^{\prime} \rightarrow=C a \rightarrow+D s \rightarrow,
\end{aligned}
$$

where $C$ is given by equation $(27)_{2} ; a \rightarrow \times s \rightarrow$ is an invariant vector for refraction. Its direction is perpendicular to the incident plane; its length $p$ is the length of the perpendicular dropped from the center to the incident (refracted) ray, multiplied by the corresponding refractive index.

We have

$$
p^{2}=(a \rightarrow \times s \rightarrow)^{2}=a \rightarrow^{2} s^{2}-(a \rightarrow \cdot s \rightarrow)^{2}=2 n^{2} u_{1}-u_{2}^{2} .
$$

Equation (31) now gives

$$
\begin{aligned}
s^{\prime} \rightarrow \times s \rightarrow & =C(a \rightarrow \times s \rightarrow), \\
s^{\prime} \rightarrow \cdot s \rightarrow & =C a \rightarrow \cdot s \rightarrow+D s^{\rightarrow 2} .
\end{aligned}
$$

If $\delta$ is the angle between $s$ and $s^{\prime}$ (the angle of deviation), equations (33) are equivalent to

$$
n n^{\prime} \sin \delta=C p, \quad n n^{\prime} \cos \delta=C u_{2}+D n^{2},
$$

or

$$
C=\frac{n n^{\prime} \sin \delta}{p}, \quad D=\frac{1}{n^{2}}\left\{n n^{\prime} \cos \delta-\frac{n n^{\prime} \sin \delta}{p} u_{2}\right\}
$$

Our remaining problem is to express $\delta$ as a function of $p$, and then, by using (32), as a function of $u_{1}$ and $u_{2}$. Equation (27) gives

$$
C r \rightarrow 2=s^{\prime} \rightarrow \cdot r \rightarrow-s \rightarrow \cdot r \rightarrow,
$$

or, because of $(30)$,

$$
\begin{aligned}
C & =(1 / r)\left\{\left(n^{\prime 2}-(p / r)^{2}\right)^{1 / 2}-\left(n^{2}-(p / r)^{2}\right)^{1 / 2}\right\} \\
\sin \delta & =\left(p / n n^{\prime} r\right)\left\{\left(n^{\prime 2}-(p / r)^{2}\right)^{1 / 2}-\left(n^{2}-(p / r)^{2}\right)^{1 / 2}\right\} \\
\cos \delta & =\left(p^{2} / n n^{\prime} r^{2}\right)+\frac{1}{n n^{\prime}}\left(\left[n^{\prime 2}-(p / r)^{2}\right]\left[n^{2}-(p / r)^{2}\right]\right)^{1 / 2}
\end{aligned}
$$

Inserting (37) in (34) gives $C$ and $D$ as functions of $p$ and $u_{2}$, and therefore, because of (32), as functions of $u_{1}$ and $u_{2}$.

To solve the reverse problem, that is, to calculate $u_{1}$ and $u_{2}$ as functions of $C$ and $D$, we proceed as follows:

Equation (37) gives

$$
\left(\cos \delta-p^{2} / n n^{\prime} r^{2}\right)^{2}=\left(1 / n n^{\prime}\right)^{2}\left(n^{\prime 2}-p^{2} / r^{2}\right)\left(n^{2}-p^{2} / r^{2}\right),
$$




$$
\left(n n^{\prime} \sin \delta\right)^{2}+\left(p^{2} / r^{2}\right)\left(2 n n^{\prime} \cos \delta-n^{2}+n^{\prime 2}\right)=0 ;
$$

or, considering $(34)_{1}$

$$
2 n n^{\prime} \cos \delta=n^{2}+n^{\prime 2}-C^{2} r^{2} .
$$

Inserting this in (34) $)_{2}$, we obtain

$$
u_{2}=\frac{n^{2}+n^{\prime 2}-C^{2} r^{2}}{2 C}-\frac{D}{C} n^{2} .
$$

Equation (34) gives

$$
\begin{aligned}
p^{2}=\left(\frac{n n^{\prime} \sin \delta}{C}\right)^{2} & =\frac{n^{2} n^{\prime 2}}{C^{2}}-\left(\frac{n^{2}+n^{\prime 2}-C^{2} r^{2}}{2 C}\right)^{2}=2 n^{2} u_{1}-u_{2}^{2} \\
& =2 n^{2} u_{1}-\left(\frac{n^{2}+n^{\prime 2}-C^{2} r^{2}}{2 C}-\frac{D}{C} n^{2}\right)^{2}
\end{aligned}
$$

which gives

$$
\begin{aligned}
& u_{1}=-D r^{2} / 2+\frac{1-D}{2 C^{2}}\left(n^{\prime 2}-n^{2} D\right) \\
& u_{2}=-C r^{2} / 2+\frac{n^{2}(1-D)+\left(n^{\prime 2}-n^{2} D\right)}{2 C}
\end{aligned}
$$
(26)

We see that $u_{1}$ and $u_{2}$ are rational functions of $C$ and $D$, fulfilling equation

Equations (42) are very valuable for calculating the coefficients of $C$ and $D$, written as a series in $u_{1}$ and $u_{2}$.

\section{ThE PLANE SURFACE}

The equations for refracting a ray at a plane surface are very simple. We let the $z$ - and $z^{\prime}$-axes coincide with the axis of the system, and place the origins at the intersection point of the axis with the plane surface. Applying the refraction law here gives

$$
\begin{array}{ll}
x^{\prime}=x, & \xi^{\prime}=\xi, \\
y^{\prime}=y, & \eta^{\prime}=\eta .
\end{array}
$$

This means that the transformation is the identical transformation.

\section{TRANSITION FORMULAE}

To obtain the formulae for tracing a system of rays through a system of centered lenses, we must also know how to find, from the coordinates of the intersection point of the image ray with the reference plane through the 
center of one surface, the coordinates of the ray intersection in the reference plane through the center of the succeeding surface. We call the distance between the two centers $m$. From analytical geometry, we get

$$
\begin{array}{rlrl}
x^{\prime} & =x+\frac{m}{\left(1-2 u_{3} / n^{2}\right)^{1 / 2}} \xi, & \xi^{\prime}=\xi, \\
y^{\prime}=y+\frac{m}{\left(1-2 u_{3} / n^{2}\right)^{1 / 2}} \eta, & \eta^{\prime}=\eta .
\end{array}
$$

In tracing rays, we can use the formulae described in the preceding section. We can simplify the calculation since, instead of tracing $x, x^{\prime}, y^{\prime}$ and $y$, it proves to be sufficient to trace $u_{1}, u_{2}$, and $u_{3}$, because the functions depend only upon these variables. Moreover, for any optical image formation, we have the relation

$$
u_{1}^{\prime} u_{3}^{\prime}-u_{2}^{\prime 2}=\left(x^{\prime} \eta^{\prime}-y^{\prime} \xi^{\prime}\right)^{2}=(x \eta-y \xi)^{2}=u_{1} u_{3}-u_{2}^{2},
$$

which follows directly from (1). The actual ray tracing formulae will be published later.

\section{IMAGE ERROR THEORY}

Finally, let us sketch briefly how the image error theory can be derived, using this new method of approach.

Let us develop $A, B, C$, and $D$ as functions of $u_{1}, u_{2}$, and $u_{3}$, into a series and inspect the equations for small values of $u_{1}, u_{2}$, and $u_{3}$.

If we assume $u_{1}, u_{2}$, and $u_{3}$ to be negligible, we obtain Gaussian optics, within the realm of which $A, B, C$, and $D$ can be regarded as constant values. We have

$$
\begin{array}{ll}
x^{\prime}=A_{0} x+B_{0} \xi, & \xi^{\prime}=C_{0} x+D_{0} \xi, \\
y^{\prime}=A_{0} y+B_{0} \eta, & \eta^{\prime}=C_{0} y+D_{0} \eta
\end{array}
$$

with $(8)_{2}$

$$
A_{0} D_{0}-B_{0} C_{0}=1 \text {. }
$$

The special case, that object and image are in optically conjugated points, is indicated by

$$
B_{0}=0 \text {. }
$$

Then $D_{0}=1 / A_{0}$, and

$$
\begin{array}{ll}
x^{\prime}=A_{0} x, & \xi^{\prime}=C_{0} x+\left(1 / A_{0}\right) \xi, \\
y^{\prime}=A_{0} y, & \eta^{\prime}=C_{0} y+\left(1 / A_{0}\right) \eta .
\end{array}
$$

From (48) and (46) can be derived all the laws of Gaussian optics. 
Let us now consider the linear members of $A, B, C$, and $D$, but neglect all higher orders. This leads to the so-called Seidel theory of aberrations. We have again

$$
D=\frac{1+B C}{A},
$$

but the nine first-order derivatives of $A, B$, and $C$ are not independent. Equations (9) show that between these derivatives at $u_{1}=u_{2}=u_{3}=0$ there exist the three equations

$$
\begin{aligned}
\frac{B_{0}}{A_{0}}\left(A_{0} C_{1}-C_{0} A_{1}\right)-\left(A_{0} C_{2}-C_{0} A_{2}\right) & =\frac{A_{1}}{A_{0}} \\
\frac{B_{0}^{2}}{A_{0}}\left(A_{0} C_{1}-C_{0} A_{1}\right)-\left(A_{0} C_{3}-C_{0} A_{3}\right) & =\frac{B_{0} A_{1}+B_{1} A_{0}}{A_{0}^{2}}, \\
\frac{B_{0}^{2}}{A_{0}^{2}}\left(A_{0} C_{2}-C_{0} A_{2}\right)-\frac{B_{0}}{A_{0}}\left(A_{0} C_{3}-C_{0} A_{3}\right) & =\frac{B_{0} A_{2}-B_{2} A_{0}}{A_{0}^{2}}-\frac{A_{3}}{A_{0}} .
\end{aligned}
$$

From these equations, we get

$$
\begin{aligned}
& B_{0}\left(B_{1}-A_{2}\right)=A_{0}\left(B_{2}-A_{3}\right), \\
& A_{0} C_{2}-C_{0} A_{2}=\frac{B_{0}}{A_{0}}\left(A_{0} C_{1}-C_{0} A_{1}\right)-\frac{A_{1}}{A_{0}} \\
& A_{0} C_{3}-C_{0} A_{3}=\frac{B_{0}^{2}}{A_{0}}\left(A_{0} C_{1}-C_{0} A_{1}\right)-\frac{B_{0} A_{1}-B_{1} A_{0}}{A_{0}^{2}} .
\end{aligned}
$$

Let us now put the origin at the Gaussian image point, so that $B_{0}=0$, and equations (51) simplify to

$$
\begin{aligned}
B_{2} & =A_{3}, \\
A_{0} C_{2}-C_{0} A_{2} & =-\frac{A_{1}}{A_{0}}, \\
A_{0} C_{3}-C_{0} A_{3} & =-\frac{B_{1}}{A_{0}} .
\end{aligned}
$$

Substituting this in the first equation of (1), we find, after rearranging, that

$$
\begin{aligned}
& x^{\prime}-A_{0} x=\left(A_{1} u_{1}+A_{2} u_{2}+A_{3} u_{3}\right) x+\left(B_{1} u_{1}+B_{2} u_{2}+B_{3} u_{3}\right) \xi \\
& y^{\prime}-A_{0} y=\left(A_{1} u_{1}+A_{2} u_{2}+A_{3} u_{3}\right) y+\left(B_{1} u_{1}+B_{2} u_{2}+B_{3} u_{3}\right) \eta
\end{aligned}
$$


Geometrical inspection will show that the five quantities $A_{1}, A_{2}, A_{3}=B_{2}$, $B_{1}$, and $B_{3}$ correspond to the five image errors:

$B_{3}$ represents the spherical aberration;

$A_{3}=B_{2}$ represents the coma error;

$A_{2}$ and $B_{1}$ represent the field errors;

$A_{1}$ represents the distortion.

In (53) the coordinates $x^{\prime}, y^{\prime}$ of the intersections of the image ray with the image plane are given as functions of $x, y, \xi, \eta . x=y=0$ characterizes the axis point of the object, $\xi=\eta=0$ characterizes the infinite point. We can say, therefore, that $A_{1}, A_{2}, A_{3}, B_{1}, B_{2}, B_{3}$ give us the image errors of our object for a stop at infinity. To obtain the image errors for a finite stop, we have to replace $\xi, \eta$, by the coordinates, $x_{p}, y_{p}$, of the intersection point of our ray with the plane of the diaphragm. Within the region of validity of the Seidel theory, we get a simple linear transformation. If $k$ is the distance between object and stop, we have

$$
\begin{array}{lll}
x_{p}=x+k \xi, & & \xi=\frac{x_{p}-x}{k}, \\
& \text { or } & \\
y_{p}=y+k \eta, & \eta=\frac{y_{p}-y}{k} .
\end{array}
$$

Inserting (54) into (53) we find $x^{\prime}$ and $y^{\prime}$ as functions of $x, y, x_{p}, y_{p}$, the coefficients being the image errors for finite stop.

The method developed in this paper allows one to obtain the image coordinates in a rotation symmetric optical system as functions of the object coordinates by a series of substitutions. The only other general method having Hamilton's characteristic function leads to an elimination problem, hitherto unsolved.

Hamilton's method is more elegant since it uses only a single function to describe an optical instrument; the method of this paper leads to four functions connected by three differential equations. However, an explicit way was found to construct our function for any given system of centered lenses, whereas the characteristic function of Hamilton is explicitly known only for a single refracting surface or a plane parallel plate. Thus, the new method seems to be more adaptable to practical problems.

The last paragraph tries to show that the access to the image theory by the direct method is as simple as it is by using Hamilton's characteristic function.

ROCHESTER, N. Y. 